\title{
ChApter
}

\section{Synthesis, Modification and Turnover of Proteins during Aging}

\author{
Suresh I.S. Rattan*
}

\begin{abstract}
A

lterations in the rate and extent of protein synthesis, accuracy, posttranslational modifications and turnover are among the main molecular characteristics of aging. A decline in the cellular capacity through proteasomal and lysosomal pathways to recognize and preferentially degrade damaged proteins leads to the accumulation of abnormal proteins during aging. The consequent increase in molecular heterogeneity and impaired functioning of proteins is the basis of several age-related pathologies, such as cataracts, sarcopenia and neurodegerative diseases. Understanding the proteomic spectrum and its functional implications during aging can facilitate developing effective means of intervention, prevention and therapy of aging and age-related diseases.
\end{abstract}

\section{Introduction}

A decline in the rate of total protein synthesis is one of the most commonly observed age-associated biochemical changes in cells, tissues, organs and organisms, including human beings. The implications and consequences of slower rates of protein synthesis are manifold in the context of aging and age-related pathology. Although there is a considerable variability among different tissues and cell types in the extent of decline (varying from $20 \%$ to $80 \%$ ), the fact remains that the bulk protein synthesis slows down during aging. ${ }^{1,2}$ However, it should be pointed out that age-related slowing down of bulk protein synthesis does not mean that the synthesis of each and every protein becomes slower uniformly during aging. Furthermore, even though bulk protein synthesis slows down with age, total protein content of the cell generally increases because of an accumulation of abnormal proteins during aging. Age-related changes in protein synthesis are regulated both at the transcriptional and pretranslational levels in terms of the availability of individual mRNA species for translation, ${ }^{2}$ and at the translational and posttranslational levels in terms of alterations in the components of the protein synthetic machinery and the pattern of postsynthetic modifications that determine the activity, specificity and stability of a protein.

The aim of this article is to provide an overview of the regulation and misregulation, synthesis, modifications and turnover of proteins, including the role of protein errors and accumulation of abnormal proteins during aging.

\section{Efficiency and Accuracy of Protein Synthesis during Aging}

Eukaryotic protein synthesis is a highly complex process, which requires about 200 small and large components to function effectively and accurately in order to translate one mRNA molecule, while using large quantities of cellular energy. There are three major components of the

*Suresh Rattan—Laboratory of Cellular Aging, Department of Molecular Biology, Aarhus University, DK8000 Aarhus—C, Denmark. Email: rattan@mb.au.dk

Protein Metabolism and Homeostasis in Aging, edited by Nektarios Tavernarakis. (C)2009 Landes Bioscience and Springer Science+Business Media. 
translational apparatus: (1) the translational particle, the ribosome; (2) the amino acid transfer system or charging system; and (3) the translational factors. ${ }^{3}$ The protein-synthesizing apparatus is highly organised and its macromolecular components are not freely diffusible within cells. The rate and accuracy of protein synthesis can be critical for aging. Since, the error frequency of amino acid misincorporation is generally considered to be quite high $\left(10^{-3}\right.$ to $\left.10^{-4}\right)$ as compared with nucleotide misincorporation, the role of protein error feedback in aging has been a widely discussed issue. ${ }^{4,5}$

So far, no direct estimates of protein error levels in any aging system have been made primarily due to the lack of appropriate methods to determine spontaneous levels of error in a normal situation. However, several indirect estimates of the accuracy of translation in cell-free extracts, using synthetic templates or natural mRNAs have been made. Studies performed on animal tissues, such as chick brain, mouse liver and rat brain, liver and kidney, did not reveal any major age-related differences in the capacity and accuracy of ribosomes to translate poly $(\mathrm{U})$ in cell-free extracts. ${ }^{6}$ However, these attempts to estimate the error frequencies during in vitro translation of $\operatorname{poly}(\mathrm{U})$ template were inconclusive because the error frequencies encountered in the assays were several times greater than the estimates of natural error frequencies. Another indirect method that has been used to detect misincorporation of amino acids during aging is the method of two-dimensional (2D) gel electrophoresis of proteins, but no age-related increase in amino acid misincorporation affecting the net charge on proteins was observed in histidine-starved human fibroblasts and in nematodes. In contrast to this, using mRNA of CcTMV coat protein for translation by cell extracts prepared from young and old human fibroblasts, a seven-fold increase in cysteine misincorporation during cellular aging has been observed. ${ }^{7,8}$ These studies also showed that an aminoglycoside antibiotic paromomycin $(\mathrm{Pm})$, which is known to reduce ribosomal accuracy during translation in vivo and in vitro, induces more errors in the translation of CcTMV coat protein mRNA by cell extracts prepared from senescent human fibroblasts than those from young cells. Indirect evidence that indicates the role of protein errors in cellular aging can be drawn from studies on the increase in the sensitivity of human fibroblasts to the life-shortening and aging-inducing effects of Pm and another aminoglycoside antibiotic G418., ${ }^{910}$ Further evidence in support of the role of errors comes from experiments which showed that an induction and increase in protein errors can accelerate aging in human cells and bacteria. ${ }^{1,2,4,11,12}$ Similarly, an increase in the accuracy of protein synthesis can slow aging and increase the lifespan in fungi. ${ }^{13,14}$ Although a global "error catastrophe" as a cause of aging due to errors in each and every macromolecule is considered unlikely, it is not ruled out that some kind of errors in various components of protein synthetic machinery, including tRNA charging, may have long-term effects on cellular stability and survival.

\section{Altered Protein Synthesis during Aging}

The translational process can be envisaged to proceed in three steps-initiation, elongation and termination, followed by posttranslational modifications, including folding, which give the protein a functional tertiary structure. The initiation step is considered to be the main target for the regulation of protein synthesis during cell cycle, growth, development, hormonal response and under stress conditions including heat shock, irradiation and starvation. ${ }^{15,16} \mathrm{With}$ respect to aging, however, the rate of initiation appears to remain unaltered. For example, using in vitro assays, the conversion of isolated $40 \mathrm{~S}$ and $60 \mathrm{~S}$ ribosomal subunits into the $80 \mathrm{~S}$ initiation complex has been reported to decrease by less than $15 \%$ in old Drosophila, rat liver and kidney and mouse liver and kidney. On the other hand, since polysomal fraction of the ribosomes decreases during aging, it implies that the activity of an anti-ribosomal-association factor eIF-3 may increase during aging. The activity of eIF-2, which is required for the formation of the ternary complex of Met-tRNAi, GTP and eIF-2, has been reported to decrease in rat tissues during development and aging. Attenuation of hypusine formation on eIF-5A during senescence of human diploid fibroblasts has been reported. ${ }^{17}$ Recently, a novel role of eIF-5A in protein chain elongation has been reported, ${ }^{18}$ which may also be important in relation to aging. Similar studies on other eIFs and in other aging systems are yet to be performed and it is necessary that detailed studies on eIFs 
are also undertaken in the context of aging and the question of the regulation of protein synthesis at the level of initiation is reinvestigated.

Several studies have been performed on age-related changes in the number of ribosomes, thermal stability, binding to aminoacyl-tRNA, the level of ribosomal proteins and rRNAs, sensitivity to aminoglycoside antibiotics and the fidelity of ribosomes. ${ }^{6}$ Although there is a slight decrease in the number of ribosomes in old animals, this does not appear to be a rate-limiting factor for protein synthesis due to ribosomal abundance in cells. Instead, several studies indicate that the biochemical and biophysical changes in ribosomal characteristics may be more important for translational regulation during aging. For example, the ability of aged ribosomes to translate synthetic poly $(U)$ or natural globin mRNA decreases significantly. A decrease in the translational capacity of ribosomes has also been observed in rodent tissues such as muscle, brain, liver, lens, testis and parotid gland and in various organs of Drosophila. 19,20 $^{2}$

The reasons for the functional changes observed in aging ribosomes are not known at present. Some attempts have been made to study the effect of aging on rRNAs and ribosomal proteins. Although a three-fold increase in the content of rRNA has been reported in late passage senescent human fibroblasts, it is not clear whether the quantity and quality of individual rRNA species undergo alterations during aging and what effect such a change might have on ribosome function. Similarly, although an increase in mRNA levels for ribosomal protein L7 has been reported in aged human fibroblasts and rat preadipocytes, there are no differences in the electrophoretic patterns of the ribosomal proteins in young and old Drosophila and mouse liver. ${ }^{6}$

The formation of the $80 \mathrm{~S}$ initiation complex is followed by the repetitive cyclic event of peptide chain elongation, a series of reactions catalysed by elongation factors (EFs; also abbreviated as eEFs). ${ }^{21,22}$ Various estimates of the elongation rates in eukaryotic cells give a value in the range of 3 to 6 amino acids incorporated per ribosome per second, which is several times slower than the prokaryotic elongation rate of 15 to 18 amino acids incorporated per second. ${ }^{23}$ With regard to aging, a slowing-down of the elongation phase of protein synthesis has been suggested to be crucial in bringing about the age-related decline in total protein synthesis. This is because a decline of up to $80 \%$ in the rate of protein elongation has been reported by estimating the rate of phenylalanyl-tRNA binding to ribosomes in poly $(\mathrm{U})$-translating cell-free extracts from old Drosophila, nematodes and rodent organs. ${ }^{24}$ In vivo, a two-fold decrease in the rate of polypeptide chain elongation in old WAG albino rat liver and brain cortex has been reported. Similarly, a decline of $31 \%$ in the rate of protein elongation in the livers of male Sprague-Dawley rats has been reported, by measuring the rate of polypeptide chain assembly which was 5.7 amino acids per second in young animals and was 4.5 amino acids per second in old animals. ${ }^{25}$ However, these estimates of protein elongation rates have been made for "average" size proteins. It will be important to see if there is differential regulation of protein elongation rates for different proteins during aging.

The elongation of polypeptide chain is mediated by 2 elongation factors, eEF-1 and eEF-2 in eukaryotes (a third factor, EF-3, is reported only in yeast), which are highly conserved during evolution. ${ }^{21}$ The activity of eEF-1 declines with age in rat livers and Drosophila and the drop parallels the decrease in protein synthesis. ${ }^{6,20}$ This decline in the activity of eEF-1 has been correlated only to EF-1A as no changes were observed in the EF-1beta-gamma-mediated activity. Using more specific cell-free stoichiometric and catalytic assays, a 35-45\% decrease in the activity and amounts of active eEF-1A has been reported for serially passaged senescent human fibroblasts, old mouse and rat livers and brains. ${ }^{6,20,26}$

In the case of eEF-2 that catalyses the translocation of peptidyl-tRNA on the ribosome during the elongation cycle, conflicting data are available regarding the changes during aging. For example, a lack of difference in the rate of translocation has been observed during the translation of poly $(\mathrm{U})$ by cell-free extracts prepared from young and old Drosophila and from rodent organs..$^{27,28}$ Similarly, although the proportion of heat-labile eEF-2 increases during aging, the specific activity of eEF-2 purified from old rat and mouse liver remains unchanged. ${ }^{29}$ In contrast, a decline of more than $60 \%$ in the amount of active eEF-2 has been reported during aging of human fibroblasts in culture, measured by determining the content of diphtheria toxin-mediated ADP-ribosylatable 
eEF-2 in cell lysates. ${ }^{30}$ However, using the same assay, no age-related change in the amount of ADP-ribosylatable eEF-2 was detected in rat livers. ${ }^{31}$ Increased fragmentation of eEF-2 due to oxidation has also been reported in old rat livers. ${ }^{32}$ Acidic variants of eEF-2 in isolated rat heart and cultured cardiomyocytes have also been reported. ${ }^{33}$ Further studies are required to determine if there are any qualitative and quantitative changes in eEF-2 at the levels of transcription, translation and posttranslational modifications and how such changes are related with the regulation of protein synthesis during aging.

The cycle of peptide chain elongation continues until one of the three stop codons is reached. There is no aa-tRNA complementary to these codons and instead a termination factor or a release factor (RF) binds to the ribosome and induces the hydrolysis of both the aminoacyl linkage and the GTP, releasing the completed polypeptide chain from the ribosome. Studies in aging Drosophila and old rat livers and kidneys have shown that the release of ribosome bound $\mathrm{N}$-formylmethionine, a measure of the rate of translation termination, was not affected with age. ${ }^{28}$ Direct estimates of the activity of the termination factor during aging have not been yet made.

\section{Postranslational Modifications during Aging}

Accurate translation of mRNA, followed by appropriate modifications of the polypeptide chain, is essential for its normal folding, targeting and specificity. A misregulation in any of these steps can have far reaching biological consequences, including its effects on cell growth, division and survival. A large number of posttranslational modifications of proteins have been described that determine the activity, stability, specificity, transportability and lifespan of a protein. Several of these modifications are highly specific and regulated involving various enzymatic pathways, but there are several nonenzymatic modifications of proteins which occur stochastically but depend on the ternary structures. ${ }^{34,35}$

\section{Phosphorylation}

Phosphorylation of serine, threonine and tyrosine residues is one of the best studied modifications of proteins. The coordinated activities of protein kinases, which catalyse phosphorylation and of protein phosphatases which catalyse dephosphorylation, regulate several biological processes, including protein synthesis, cell division, signal transduction, cell growth and development. ${ }^{36}$ Altered pattern of protein phosphorylation may be one of the reasons for age-related alterations in protein function and activity and can be a major cause of the failure of homeodynamics and aging. For example, inhibition of DNA synthesis and the loss of proliferative capacity is the ultimate characteristic of normal diploid cells undergoing aging in vitro. ${ }^{37}$ Although several putative inhibitors of DNA synthesis have been identified in senescent cells, little is known about the mechanisms of action and the regulation of activity of these inhibitors. It is possible that the activity of several of these inhibitors is regulated by phosphorylation. For example, several studies have shown age-related alterations in cell-cycle-regulated gene expression of various genes such as $c-f o s$, $c$-jun, JunB, c-myc, c-Ha-ras, $p 53, c d c 2, c y c A, c y c B, c y c D$ and retinoblastoma gene $R B 1$. Although phosphorylation is involved in regulating the activities of the gene products of almost all these genes, a decrease in phosphorylated cyclin $\mathrm{E}$ and $\mathrm{Cdk} 2$ and failure to phosphorylate $R B 1$ gene product $\mathrm{p} 110^{\mathrm{Rb}}$ and $c d c 2$ product $\mathrm{p} 34^{\mathrm{cdc} 2}$ during cellular aging have been reported at present. ${ }^{38}$ It will be important to find out if there are age-related alterations in the phosphorylation state of other cell cycle related gene products, proteins involved in DNA and RNA synthesis, including various transcription factors..$^{39,40}$

Various components of the protein synthetic apparatus undergo phosphorylation and dephosphorylation and thus regulate the rates of protein synthesis. ${ }^{23}$ For example, phosphorylation of eIF-2 correlates with inhibition of initiation reactions and consequently the inhibition of protein synthesis. Conditions like starvation, heat shock and viral infection, which inhibit the initiation of protein synthesis, induce the phosphorylation of eIF-2 in various cells. Stimuli such as insulin and phorbol esters modulate the phosphorylation of eIF-3, eIF-4B and eIF- $4 \mathrm{~F}$ by activating various protein kinases. ${ }^{23}$ Since the activity of eIF- 2 has been reported to decrease during aging, it is 
possible that the phosphorylation status of eIF-2 also changes during aging. However, no studies are available on age-related changes in the phosphorylation pattern of initiation factors.

At the level of protein elongation, the phosphorylation of elongation factors eEF-1A and eEF-2 appears to be involved in regulating their activities. It will be important to see whether the age-related decline in the activities of elongation factors is accompanied by a parallel change in the extent of phosphorylation of these enzymes. Incidentally, it has been reported that there is an increase in the levels of phosphorylated eEF-1 and eEF-2 during mitosis when minimal protein synthesis occurs. Furthermore, there is indirect evidence that alterations in the phosphorylation and dephosphorylation of eEF-2 due to changes in the activities of eEF-2-specific protein kinase III, ${ }^{41}$ and PP2A phosphatase ${ }^{42}$ may affect the rates of protein synthesis during aging in rat livers.

Phosphorylation also occurs in other proteins that participate in the translational process. For example, the regulatory role of phosphorylation of aa- $t$ RNA synthetase in protein synthesis has been suggested. ${ }^{43}$ However, to what extent the decline in the activity and the accumulation of heat-labile aa-tRNA synthetases reported in studies performed on various organs of aging mice and rats is related to their phosphorylation is not known. Furthermore, since the phosphorylation of the S6 ribosomal protein correlates with the activation of protein synthesis, failure to phosphorylate $S 6$ protein in senescent human fibroblasts in response to serum, ${ }^{44}$ can be one of the reasons for the decline in the rate of protein synthesis observed during aging.

Pathways of intracellular signal transduction depend on sequential phosphorylation and dephosphorylation of a wide variety of proteins. All phosphorylation reactions result from the action of one or more kinases and the ratio between two interconvertible forms of kinases (active and inactive) acts as a control mechanism for many cellular functions. Studies performed on aging cells have not shown any deficiency in the amount, activity or ability of protein kinase-C (PKC) to elicit signalling pathway. ${ }^{45}$ There is also evidence that senescent human fibroblasts retain their ability to phosphorylate proteins in the PKC signal transduction pathway. It appears that the PKCs are largely unaltered in fibroblasts, although the body of information about phosphorylation mechanisms is still very limited. ${ }^{39}$

Growth factor receptors for EGF, FGF, PDGF, insulin, glucocorticoids and several other hormones also possess protein kinase activity. Therefore, deficiencies in the phosphorylation process of receptors would be a logical explanation for the age-related decline of responsiveness to hormonal action and growth stimulation. However, there is no age-related decline in the autophosphorylation activity of various growth factor receptors. ${ }^{46,47}$ Similarly, most of the PKC-mediated pathways of intracellular signal transduction in response to various mitogens including phorbol esters appear to remain unaltered in senescent fibroblasts. ${ }^{46-48}$ However, a decline in both serine/ threonine- and tyrosine-specific protein kinase signals after activation has been observed in the case of T-lymphocytes in aging mice. ${ }^{49}$ Similarly, alterations in MAPK phosphorylation have been observed in rodents, ${ }^{50}$ while $\mathrm{PKC}$ phowsphorylation changes are seen in human pathological aging, including Alzheimer's disease and neurodegenerative processes. ${ }^{51}$.

\section{Oxidation}

It is often observed that inactive and abnormal proteins accumulate in old cells and tissues. This increased amount of debris in the cytoplasm can be inhibitory for cell growth and normal metabolism and thus contribute towards failure of homeostasis. One of the reasons for the inactivation of enzymes can be their oxidative modification by oxygen free radicals and by mixed-function oxidation (MFO) systems or metal catalyzed oxidation (MCO) systems. Since some amino acid residues, particularly proline, arginine and lysine, are oxidized to carbonyl derivatives, the amount of carbonyl content of proteins has been used as an estimate of protein oxidation during aging. . $2-56^{-0}$

An increase in the levels of oxidatively modified proteins has been reported in old human erythrocytes of higher density and in cultured human fibroblasts from normal old donors and from individuals suffering from progeria and Werner's syndrome. ${ }^{57}$ Similarly, there was a two-fold increase in the protein carbonyl content of brain proteins of retired breeder Mongolian gerbils, which was reversed by treatment with the spin-trapping compound $\mathrm{N}$-tert-butyl-phenylnitrone. ${ }^{58}$ 
An age-related increase in the carbonyl content has also been reported in houseflies, fruitflies, nematodes and mouse organs. ${ }^{59-63}$

The loss of activity of 6-phosphogluconate dehydrogenase and liver malic enzyme during aging is related to the loss of lysine and histidine residues by oxidation. ${ }^{64}$ Oxidation of a cysteine residue in glyceraldehyde-3-phosphate dehydrogenase may be responsible for its inactivation during aging in rat muscles. ${ }^{65}$ It has also been reported that the concentration of the oxidation products of human lens proteins and skin collagen increases along with the accumulation of oxidative forms of alpha-crystallin in patients with age-related cataract. ${ }^{66}$ However, the content of ortho-tyrosine and dityrosine, formed by the oxidation of phenylalanine and tyrosine, respectively, did not increase in the aging human lens. ${ }^{67}$ Structural alterations introduced into proteins by oxidation can lead to aggregation, fragmentation, denaturation and distortion of secondary and tertiary structure, thereby increasing the proteolytic susceptibility of oxidized proteins. Furthermore, toxic products of carbonyl modifications can react with other macromolecules and affect various metabolic processes.

Generally it is thought that there are no systems for the repair of oxidatively damaged proteins and these must be degraded to avoid their accumulation. However, at least one kind of protein repair system has been identified. For example, oxidatively-induced disulphide bridges in cysteine and methionine lead to the formation of sulfenic acid, sulfinic acid and methionine sulphoxide which can accumulate during aging. These damages can be repaired by the methyl-sulfoxide reductase (MSR) system comprising of at least 3 Msr enzymes whose activity is decreased during aging. ${ }^{68,69}$ Senile graying of human hair due to increased oxidative stress is accompanied by a reduction of the activities of MSR system. ${ }^{70}$ In contrast to this, overexpression of one of the Msr genes increases the lifespan of Drosophila and improves their stress tolerance to oxidative damage-inducing agents. ${ }^{71}$

\section{Glycation}

Glycation is one of the most prevalent covalent modifications in which the free amino groups of proteins react with glucose to form a ketoamine called Amadori product. This is followed by a sequence of further reactions and rearrangements producing the so-called advanced glycosylation end products (AGEs). ${ }^{72,73}$ Most commonly, it is the long-lived structural proteins such as lens crystallins, collagen and basement membrane proteins which are more susceptible to glycation. In the case of skin, vimentin has been found to be as the main protein becoming glycated during aging. ${ }^{74}$ The glycated proteins are then more prone to form crosslinks with other proteins, leading to structural and functional alterations. ${ }^{5}$

An increase in the levels of glycated proteins during aging has been observed in a wide variety of systems. For example, there is an increase in the level of glycated lysine residues of rat sciatic nerve, aorta and skin collagen during aging. ${ }^{75}$ There is an increase in the glycation of human collagen and osteocalcin during aging. ${ }^{76}$ The formation and the accumulation of the AGEs are implicated in the physiology and pathology of senescence. It has been observed that pentosidine (cross-linked glycated lysine and arginine), carboxylmethyllysine (CML, glycated and oxidated proteins) and pyrroline increase with age in normal and diabetic humans. ${ }^{77}$ By using AGE-specific antibodies, an AGE-modified form of human hemoglobin has been identified, whose levels increase during aging and in patients with diabetes-induced hyperglycemia. ${ }^{78}$

\section{Deamidation, Racemization and Isomerization}

Age-related changes in the catalytic activity, heat stability, affinity for substrate and other physical characteristics, such as the conformation of proteins may also be due to the charge change introduced by conversion of a neutral amide group to an acidic group by deamidation. Spontaneous deamidation of asparaginyl and glutaminyl residues of several proteins has been related with the observed accumulation of their inactive and heat labile isoforms during aging. ${ }^{65}$ The sequential deamidation of two asparagine residues of triphosphate isomerase is responsible for the differences of the isoenzymes present in aging cells and tissues, such as bovine eye lens and human skin fibroblasts from old donors and patients with progeria and Werner's syndrome. ${ }^{79}$ Deamidation of glucose-6-phosphate isomerase produces the variant of the enzyme that accumulates in aging bovine lenses. ${ }^{80}$ 
The interconversion of optical isoforms of amino acids, called racemization, has been reported to increase during aging. For example, the concentration of $\mathrm{D}$-aspartate in protein hydrolysates from human teeth, erythrocytes and eye lens increases with age. ${ }^{81}$ Racemization of tyrosine has been reported to occur in the aging brunescent human cataract lenses. ${ }^{82}$ The spontaneous prolyl cis-trans isomerization in proteins that may cause some of the so-called spontaneous conformational changes has been implicated in the age-related decline in the activity of certain enzymes. However, no definitive examples of enzymes undergoing this kind of posttranslational modification during aging are available. It is also not known to what extent the conformational changes associated with old rat muscle phosphoglycerate kinase, enolase and other enzymes are associated with racemization and isomerization.

\section{ADP-Ribosylation}

The structure and function of many proteins such as nuclear proteins topoisomerase I, DNA ligase II, endonuclease, histones $\mathrm{H} 1, \mathrm{H} 2 \mathrm{~B}$ and $\mathrm{H} 4$, DNA polymerases and cytoplasmic proteins adenyl cyclase and elongation factor eEF-2 is modulated by ADP-ribosylation. ADP-ribosylation of proteins is involved in various cellular processes such as maintenance of chromatin structure, DNA repair, protein synthesis, cell differentiation and cell transformation. ${ }^{83,84}$

Indirect evidence suggests that poly-ADP-ribosylation of proteins may decrease during aging because the activity of poly(ADP-ribose)polymerase (PARP) decreases in aging human fibroblasts both as a function of donor age and during serial passaging in vitro. ${ }^{85}$ Similarly, the direct relationship observed between maximum lifespan of a species and the activity of PARP in mononuclear leukocytes of 13 mammalian species indicates its important role in aging and longevity. ${ }^{83,86}$

One cytoplasmic protein that can be specifically ribosylated by diphtheria toxin and exotoxin $\mathrm{A}$ is the protein elongation factor eEF-2. ADP-ribosylation of the diphthamide (modified histidine 715) residue of eEF-2 results in the complete abolition of its catalytic activity. ${ }^{21}$ There is evidence that increased ADP-ribosylation of eEF-2 is correlated with cellular aging. For example, the amount of eEF-2 that can be ADP-ribosylated in the presence of diphtheria toxin in cell-free extracts decreases significantly during aging of human fibroblasts in culture..$^{30,32}$

\section{Methylation}

Methylation of nitrogens of arginine, lysine and histidine and carboxyls of glutamate and aspartate residues is a widely observed posttranslational modification that is involved in many cellular functions. Although most of our present understanding regarding the significance of protein methylation has come from studies on bacterial chemotaxis, muscle contraction, electron transport, processing of pituitary hormones and gene expression, its role in aging is beginning to emerge. ${ }^{87}$

Proteins whose activities are increased by methylation include alcohol dehydrogenase, histones, ribosomal proteins, cytochrome $\mathrm{C}$, eEF-1A, myosin, myelin and rhodopsin. Of these, decreased methylation of histones has been reported in livers and brains of aging rats. On the other hand, there is no difference in the extent of methylation of newly synthesized histones during cellular aging of human fibroblasts in culture. Studies on the levels of methylated histidine, arginine and lysine of myosin isolated from the leg muscles of aging rats, mice and hamsters showed unchanged levels of histidine, decreased levels of arginine and trimethyllysine and increased levels of monomethyllysine. .,88 $^{6}$

During the aging of erythrocytes, there is an increase in the number of methyl groups per molecule of band 2.1 (ankyrin) and band 3 protein, which correlates with increased membrane rigidity of erythrocytes during aging. ${ }^{89}$ Similarly, there is a several fold increase in the number of methyl acceptor proteins in the eye lenses from aged humans and persons suffering from cataract. ${ }^{90}$ The number of carboxylmethylatable sites of cerebral membrane-bound proteins also increases in rat brain during aging. ${ }^{91}$ At present, age-related changes in the methylation of other proteins such as ribosomal proteins, calmodulin, cytochrome $\mathrm{C}$ and myosin have not been studied. It is clear that protein methylation is involved in diverse functions including protein synthesis and turnover and that it should be studied thoroughly in relation to the process of aging. 


\section{Proteolytic Processing}

Many newly synthesised proteins undergo posttranslational proteolytic processing by which certain conformational restraint on the inactive precursor is released and a biologically active protein is generated. Several inactive precursors of enzymes called zymogens, precursors of growth factors, peptide and protein hormones such as insulin, precursors of extracellular matrix and many other secretory proteins including various proteases such as collagenase, undergo proteolytic processing. There are no systematic studies performed on age-related changes in posttranslational proteolytic processing of any proteins. However, there is some evidence that alterations in proteolytic processing may be one of the reasons for the appearance or disappearance of certain proteins during aging. For example, the appearance of the "senescent cell antigen" on the surface of a wide variety of aging cells is derived from the proteolysis of band 3 protein. ${ }^{92}$ The exposure of senescent cell-specific epitopes on fibronectin, ${ }^{93}$ may also be due to altered proteolytic processing. Progressive proteolysis of a $90 \mathrm{kDa}$ protein, Tp-90 terminin , into Tp-60 and Tp-30 terminin in senescent cells and in cells committed to apoptosis has been reported..$^{94}$

Altered proteolytic cleavage of the beta-amyloid precursor protein is well known to play an important role in the pathogenesis of Alzheimer's disease. ${ }^{95,96}$ Increased proteolysis of a conformationally more labile single-chain form of the lysosomal protease cathepsin B has been suggested as a reason for the age-related decline in its activity during aging of human fibroblasts. ${ }^{97}$ Similarly, alterations in the activity of collagenase during aging of human fibroblasts has been suggested to be due to structural and catalytic changes. ${ }^{98}$

\section{Other Modifications}

In addition to the types of posttranslational modifications mentioned above, there are some other modifications that determine the structure and function of various proteins and may have a role to play during aging. For example, the incorporation of ethanolamine into protein elongation factor eEF-1A may be involved in determining its stability and interaction with intracellular membranes. ${ }^{99}$ Whether this modification has any role in the regulation of the activity of eEF-1A during aging is not known at present. Similarly, the protein initiation factor eIF-5A contains an unusual amino acid, hypusine, which is synthesized posttranslationally as a result of a series of enzymatically catalysed alterations of a lysine residue. ${ }^{100}$ Since the absence of hypusine in eIF-5A blocks the initiation of protein synthesis, reduced activity of a hypusine synthase enzyme has been reported in senescent human fibroblasts. ${ }^{17}$

Detyrosination of microtubules affecting the cytoskeletal organization and many other cellular functions, may also be important during aging. Furthermore, the roles of chaperones in protein folding and conformational organization are yet to be studied in relation to the aging process. There is some evidence that both the pentose-mediated protein crosslinking and transglutaminase-mediated crosslinking of proteins is involved in aging. For example, there is a high correlation between pentosidine protein crosslinks and pigmentation in senescent and cataract affected human lens. ${ }^{101}$ Similarly, an increase in transglutaminase activity during cellular apoptosis, differentiation and aging of human keratinocytes indicates an important role of this modification in the process of aging. ${ }^{102}$

Protein tyrosine sulfation is another posttranslational modification that may have significance in protein alteration during aging because it is involved in determining the biological activity of neuropeptides and the intracellular transportation of a secretory protein. ${ }^{103}$ Similarly, prenylation, the covalent attachment of isoprenoid lipids on cysteine-rich proteins, is involved in the regulation of the activity of some proto-oncogenic ras proteins and the nuclear lamins A and B. ${ }^{104}$ These studies have indicated a critical role for prenylation in the regulation of oncogenesis, nuclear structure, signal transduction and cell cycle progression, functions very much related with the causative aspects of aging. There is an age-dependent decrease in the activity of prenyltransferases in the rat liver, which may account for the changes in the synthesis and turnover of mevalonate pathway lipids, including cholestrol, ubiquinone and dolichol. ${ }^{105}$ 


\section{Protein Turnover during Aging}

Efficient macromolecular turnover is integral to the normal function and survival of a biological system. Although there are large variations in the rates of degradation of individual proteins, it is generally observed that overall protein turnover slows down during aging. ${ }^{1,20}$ The physiological consequences of decreased protein turnover include the accumulation of altered and abnormal proteins in the cell, an altered pattern of posttranslational modifications due to increased dwell time and a disruption of the organisation of the cytoskeleton and extracellular matrix. ${ }^{5}$

Age-related decline in protein turnover is generally due to a decrease in the proteolytic activity of various lysosomal and cytoplasmic proteases. Molecular details of various pathways of protein degradation, such as the proteasome-mediated, ubiquitin-mediated and the lysosome-mediated pathways, are now being studied in relation to aging. ${ }^{106-108}$ For example, ubiquitin marking of proteins for degradation and ubiquitin-mediated proteolysis did not decline in aging human fibroblasts and no change in the levels of ubiquitin mRNA and ubiquitin pools was detected. ${ }^{109}$ Significantly reduced proteasomal activities during aging, with or without a parallel decrease in the amount of proteasomal components, have been reported for human fibroblasts, keratinocytes and other systems. ${ }^{69,110,111}$ The exact reasons for the age-related loss of proteasomal activities are still not known and may include the accumulation of abnormal proteasomal subunits, damaged by oxidative and glycoxidative pathways. ${ }^{12}$ Similarly, reduced lysosomal activity may be due to altered $\mathrm{pH}$ and overcrowding of the lysosomes with abnormal and damaged macromolecules such as age-pigments or lipofuscin. ${ }^{13,114}$

Other reasons for age-related changes in the activities of various proteases leading to a decrease in the rate of protein turnover include slower transcription, reduced rates of (protein) synthesis and altered pattern of postsynthetic modifications, as discussed above. Furthermore, there is evidence that certain inhibitors of various proteases, such as tissue inhibitor of metalloproteinases, ${ }^{115}$ and trypsin inhibitor, ${ }^{116}$ had increased levels of expression and activity during aging of human fibroblasts. This results in a decrease in protease activity, thus leading to decreased protein degradation during aging.

\section{Conclusion}

The synthesis, modifications and turnover of proteins are interdependent processes that practically set a limit on the efficiency of genetic information transfer from coded molecules to functional molecules. Therefore, analysing the synthesis of proteins, their modifications, which determine their activity, stability and specificity and turnover of inactive and altered proteins is central to understanding aging. Only a complete understanding of the proteomic spectrum and its functional implications with respect to normal aging can facilitate the development of effective means of intervention, prevention and therapy of aging and age-related diseases. ${ }^{117,118}$

\section{References}

1. Rattan SIS. Synthesis, modifications and turnover of proteins during aging. Exp Gerontol 1996; 31:33-47.

2. Rattan SIS. Transcriptional and translational dysregulation during aging. In: von Zglinicki T, editor. Aging at the molecular level. Dordrecht: Kluwer Acad Publ, 2003;179-91.

3. Abbott CM, Proud CG. Translational factors: in sickness and in health. Trends Biochem Sci 2004; 29:25-31.

4. Holliday R. The current status of the protein error theory of aging. Exp Gerontol 1996; 31:449-52.

5. Hipkiss A. Accumulation of altered proteins and ageing: causes and effects. Exp Gerontol 2006; 41:464-73.

6. Rattan SIS. Translation and posttranslational modifications during aging. In: Macieira-Coelho A, editor. Molecular Basis of Aging. Boca Raton, Florida: CRC Press, 1995;389-420.

7. Luce MC, Bunn CL. Altered sensitivity of protein synthesis to paromomycin in extracts from aging human diploid fibroblasts. Exp Gerontol 1987; 22:165-77.

8. Luce MC, Bunn CL. Decreased accuracy of protein synthesis in extracts from aging human diploid fibroblasts. Exp Gerontol 1989; 24:113-25.

9. Holliday R, Rattan SIS. Evidence that paromomycin induces premature ageing in human fibroblasts. Monogr Devl Biol 1984; 17:221-33. 
10. Buchanan JH, Stevens A, Sidhu J. Aminoglycoside antibiotic treatment of human fibroblasts: intracellular accumulation, molecular changes and the loss of ribosomal accuracy. Eur J Cell Biol 1987; 43:141-7.

11. Nyström T. Translational fidelity, protein oxidation and senescence: lessons from bacteria. Ageing Res Rev 2002; 1:693-703.

12. Nyström T. Aging in bacteria. Curr Opin Microbiol 2002; 5:596-601.

13. Silar P, Rossignol M, Haedens $V$ et al. Deletion and dosage modulation of the eEF1A gene in Podospora anserina: effect on the life cycle. Biogerontology 2000; 1:47-54.

14. Holbrook MA, Menninger JR. Erythromycin slows aging of Saccharomyces cerevisiae. J Gerontol Biol Sci 2002; 57A:B29-B36.

15. Dever TE. Translation initiation: adept at adapting. TIBS 1999; 24:398-403.

16. Hershey JWB, Merrick WC. The pathway and mechanism of inititation of protein synthesis. In: Sonenberg N, Hershey JWB, Mathews MB, eds. Translational Control of Gene Expression. New York: Cold Spring Harbor Laboratory Press, 2000;33-88.

17. Chen ZP, Chen KY. Dramatic attenuation of hypusine formation on eukaryotic initiation factor 5A during senescence of IMR-90 human diploid fibroblasts. J Cell Physiol 1997; 170:248-54.

18. Saini P, Eyler DE, Green R et al. Hypusine-containing protein eIF5A promotes translation elongation. Nature 2009; 459:118-21.

19. Ward W, Richardson A. Effect of age on liver protein synthesis and degradation. Hepatol 1991; 14:935-48.

20. Van Remmen H, Ward WF, Sabia RV et al. Gene expression and protein degradation. In: Masoro E, editor. Handbook of Physiology: Aging: Oxford University Press, 1995;171-234.

21. Riis B, Rattan SIS, Clark BFC et al. Eukaryotic protein elongation factors. TIBS 1990; 15:420-4.

22. Andersen GR, Nissen P, Nyborg J. Elongation factors in protein biosynthesis. Trends Biochem Sci 2003; 28:434-41.

23. Merrick WC. Mechanism and regulation of eukaryotic protein synthesis. Microbiol Rev 1992; 56:291-315.

24. Richardson A, Semsei I. Effect of aging on translation and transcription. Rev Biol Res Aging 1987; 3:467-83.

25. Merry BJ, Holehan AM. Effect of age and restricted feeding on polypeptide chain assembly kinetics in liver protein synthesis in vivo. Mech Ageing Develop 1991; 58:139-50.

26. Rattan SIS. Regulation of protein synthesis during ageing. Eur J Gerontol 1992; 1:128-36.

27. Webster GC. Protein synthesis in aging organisms. In: Sohal RS, Birnbaum LS, Cutler RG, editors. Molecular Biology of Aging: Gene Stability and Gene Expression. New York: Raven Press; 1985;263-89.

28. Webster GC. Effect of aging on the components of the protein synthesis system. In: Collatz KG, Sohal RS, editors. Insect Aging. Berlin: Springer-Verlag, 1986;207-16.

29. Takahashi R, Mori N, Goto S. Accumulation of heat-labile elongation factor 2 in the liver of mice and rats. Exp Gerontol 1985; 20:325-31.

30. Riis B, Rattan SIS, Derventzi A et al. Reduced levels of ADP-ribosylatable elongation factor-2 in aged and SV40-transformed human cells. FEBS Lett 1990; 266:45-7.

31. Rattan SIS, Ward WF, Glenting M et al. Dietary calorie restriction does not affect the levels of protein elongation factors in rat livers during ageing. Mech Ageing Develop 1991; 58:85-91.

32. Parrado J, Bougria M, Ayala A et al. Effects of aging on the various steps of protein synthesis: fragmentation of elongation factor 2. Free Rad Biol Med 1999; 26:362-70.

33. Jäger M, Holtz J, Redpath NT et al. The ageing heart: influence of cellular and tissue ageing on total content and distribution of the variants of elongation factor-2. Mech Ageing Dev 2002; 123:1305-19.

34. Soskic V, Groebe K, Schrattenholz A. Nonenzymatic posttranslational protein modifications in ageing. Exp Gerontol 2008; 43:247-57.

35. Stefani M. Protein folding and misfolding, relevance to disease and biological function. In: Smith HJ, Simons C, Seewell RDE, editors. Protein misfolding in neurodegenerative diseases: mechanisms and therapeutic strategies. Boca Raton: CRC Press, 2008;2-66.

36. Dephoure N, Zhou C, Villén J et al. A quantitative atlas of mitotic phosphorylation. Proc Natl Acad Sci USA 2008; 105:10762-7.

37. Rattan SIS. Cellular senescence in vitro. Encyclopedia of Life Sciences 2008; doi:10.1002/9780470015902. a0002567.pub2.

38. Stein GH, Dulic V. Origins of G1 arrest in senescent human fibroblasts. BioEssays 1995; 17:537-43.

39. Tresini M, Lorenzini A, Torres $C$ et al. Modulation of replicative senescence of diploid human cells by nuclear ERK signaling. J Biol Chem 2007; 282:4136-51.

40. Sedding DG. FoxO transcription factors in oxidative stress response and ageing-a new fork on the way to longevity? Biol Chem 2008; 389:279-83. 
41. Riis B, Rattan SIS, Palmquist K et al. Elongation factor 2-specific calcium and calmodulin dependent protein kinase III activity in rat livers varies with age and calorie restriction. Biochem Biophys Res Commun 1993; 192:1210-6.

42. Riis B, Rattan SIS, Palmquist K et al. Dephosphorylation of the phosphorylated elongation factor-2 in the livers of calorie-restricted and freely-fed rats during ageing. Biochem Mol Biol Int 1995; 35:855-9.

43. Meinnel T, Mechulam Y, Blanquet S. Aminoacyl-tRNA synthetases: occurrence, structure and function. In: Söll D, RajBhandary UL, editors. tRNA: Structure, Biosynthesis and Function. Washington D.C.: ASM Press, 1995;251-92.

44. Kihara F, Ninomyia-Tsuji J, Ishibashi $S$ et al. Failure in $S 6$ protein phosphorylation by serum stimulation of senescent human diploid fibroblasts, TIG-1. Mech Ageing Dev 1986; 20:305-13.

45. Blumenthal EJ, Miller ACK, Stein GH et al. Serine/threonine protein kinases and calcium-dependent protease in senescent IMR-90 fibroblasts. Mech Ageing Dev 1993; 72:13-24.

46. De Tata V, Ptasznik A, Cristofalo VJ. Effect of tumor promoter phorbol 12-myristate 13-acetate (PMA) on proliferation of young and senescent WI-38 human diploid fibroblasts. Exp Cell Res 1993; 205:261-9.

47. Farber A, Chang C, Sell C et al. Failure of senescent human fibroblasts to express the insulin-like growth factor-1 gene. J Biol Chem 1993; 268:17883-8.

48. Derventzi A, Rattan SIS, Clark BFC. Phorbol ester PMA stimulates protein synthesis and increases the levels of active elongation factors EF-1a and EF-2 in ageing human fibroblasts. Mech Ageing Dev 1993; 69:193-205.

49. Miller RA. Aging and immune function: cellular and biochemical analyses. Exp Gerontol 1994; 29:21-35.

50. Pardo VG, Facchinetti MM, Curino A et al. Age-related alteration of 1alpha,25(OH)(2)-vitamin D (3)-dependent activation of p38 MAPK in rat intestinal cells. Biogerontology 2007; 8:13-24.

51. Battaini F, Govoni S, Trabucchi M. Protein kinase C signal transmission during aging. In: Macieira-Coelho A, editor. Molecular Basis of Aging. Boca Raton: CRC Press, 1995;269-91.

52. Levine RL. Carbonyl modified proteins in cellular regulation, aging and disease. Free Rad Biol Med 2002; 32:790-6.

53. Dukan S, Farewell A, Ballesteros $M$ et al. Protein oxidation in response to increased transcriptional or translational errors. Proc Natl Acad Sci USA 2000; 97:5746-9.

54. Grune T. Oxidative stress, aging and the proteasomal system. Biogerontology 2000; 1:31-40.

55. Cloos PA, Christgau S. Post-translational modifications of proteins: implications for aging, antigen recognition and autoimmunity. Biogerontology 2004; 5:139-58.

56. Perez VI, Buffenstein R, Masamsetti V et al. Protein stability and resistance to oxidative stress are determinants of longevity in the longest-living rodent, the naked mole-rat. Proc Natl Acad Sci USA 2009.

57. Verbeke P, Clark BFC, Rattan SIS. Reduced levels of oxidized and glycoxidized proteins in human fibroblasts exposed to repeated mild heat shock during serial passaging in vitro. Free Rad Biol Med 2001; 31:1593-602.

58. Carney JM, Starke-Reed PE, Oliver CN et al. Reversal of age-related increase in brain protein oxidation, decrease in enzyme activity and loss in temporal and spatial memory by chronic administration of the spin-trapping compound N-tert-butyl-alpha-phenylnitrone. Proc Natl Acad Sci USA 1991; 88:3633-6.

59. Sohal RS, Agarwal S, Dubey A et al. Protein oxidative damage is associated with life expectancy of houseflies. Proc Natl Acad Sci USA 1993; 90:7255-9.

60. Sohal RS, Ku H-H, Agarwal S. Biochemical correlates of longevity in two closely related rodent species. Biochem Biophys Res Commun 1993; 196:7-11.

61. Beal MF. Oxidatively modified proteins in aging and disease. Free Rad Biol Med 2002; 32:797-803.

62. Goto S, Nakamura A, Radak Z et al. Carbonylated proteins in aging and excercise: immunoblot approaches. Mech Ageing Dev 1999; 107:245-53.

63. Yasuda K, Adachi H, Fujiwara Y et al. Protein carbonyl accumulation in aging dauer formation-defective (daf) mutants of Caenorhabditis elegans. J Gerontol Biol Sci 1999; 54A:B47-B51.

64. Gordillo E, Ayala A, Bautista J et al. Implication of lysine residues in the loss of enzymatic activity in rat liver 6-phosphogluconate dehydrogenase found in aging. J Biol Chem 1989; 264:17024-8.

65. Gafni A. Age-related effects in enzyme metabolism and catalysis. Rev Biol Res Aging 1990; 4:315-36.

66. Stadtman ER. Protein oxidation and aging. Science 1992; 257:1220-4.

67. Wells-Knecht MC, Huggins TG, Dyer DG et al. Oxidized amino acids in lens protein with age. Measurement of o-tyrosine and dityrosine in the aging human lens. J Biol Chem 1993; 268:12348-52.

68. Mary J, Vougier S, Picot CR et al. Enzymatic reactions involved in the repair of oxidized proteins. Exp Gerontol 2004; 39:1117-23. 
69. Petropoulos I, Conconi M, Wang X et al. Increase of oxidatively modified protein is associated with a decrease of proteasome activity and content in aging epidermal cells. J Gerontol Biol Sci 2000; 55A:B220-B7.

70. Wood JM, Decker H, Hartmann $\mathrm{H}$ et al. Senile hair graying: $\mathrm{H}_{2} \mathrm{O}_{2}$-mediated oxidative stress affects human hair color by blunting methionine sulfoxide repair. FASEB J 2009.

71. Ruan H, Tang XD, Chen ML et al. High-quality life extension by the enzyme peptide methionine sulfoxide reductase. Proc Natl Acad Sci USA. 2002; 99:2748-53.

72. Meli M, Frey J, Perier C. Native protein glycoxidation and aging. J Nutr Health Aging 2003; 7:263-6.

73. Ramasamy R, Yan SF, Schmidt AM. Methylglyoxal comes of AGE. Cell 2006; 124:258-60.

74. Kueper T, Grune T, Prahl S et al. Vimentin is the specific target in skin glycation. Structural prerequisites, functional consequences and role in skin aging. J Biol Chem 2007; 282:23427-36.

75. Oimomi M, Maeda Y, Hata F et al. A study of the age-related acceleration of glycation of tissue proteins in rats. J Gerontol 1988; 43:B98-101.

76. Miksík I, Deyl Z. Changes in the amount of e-hexosyllysine, UV absorbance and fluorescence of collagen with age in different animal species. J Gerontol 1991; 46:B111-6.

77. Lee AT, Cerami A. Role of glycation in aging. Ann NY Acad Sci 1992; 663:63-70.

78. Makita Z, Vlassara H, Rayfield E et al. Hemoglobin-AGE: a circulating marker of advanced glycosylation. Science 1992; 258:651-3.

79. Gracy RW, Yüksel KÜ, Chapman ML et al. Impaired protein degradation may account for the accumulation of "abnormal" proteins in aging cells. In: Adelman RC, Dekker EE, editors. Modifications of Proteins during Aging. New York: Alan R. Liss, 1985;1-18.

80. Cini JK, Gracy RW. Molecular basis of the isozyme of bovine glucose-6-phosphate isomerase. Arch Biochem Biophys 1986; 249:500-5.

81. Brunauer LS, Clarke S. Age-dependent accumulation of protein residues which can be hydrolyzed to D-aspartic acid in human erythrocytes. J Biol Chem 1986; 261:12538-43.

82. Luthra M, Ranganathan D, Ranganathan $S$ et al. Racemization of tyrosine in the insoluble protein fraction of brunescent aging human lenses. J Biol Chem 1994; 269:22678-82.

83. Beneke S, Alvarez-Gonzalez R, Bürkle A. Comparative characterization of poly(ADP-ribose) polymerase-1 from two mammalian species with different life span. Exp Gerontol 2000; 35:989-1002.

84. Bürkle A. Physiology and pathophysiology of poly(ADP-ribosyl)ation. BioEssays 2001; 23:795-806.

85. Dell'Orco RT, Anderson LE. Decline of poly(ADP-ribosyl)ation during in vitro senescence in human diploid fibroblasts. J Cell Physiol 1991; 146:216-21.

86. Grube K, Bürkle A. Poly(ADP-ribose) polymerase activity in mononuclear leukocytes of 13 mammalian species correlates with species-specific life span. Proc Natl Acad Sci USA 1992; 89:11759-63.

87. McBride AE, Silver PA. State of the Arg: protein methylation at arginine comes of age. Cell 2001; 106:5-8.

88. Rattan SIS, Derventzi A, Clark BFC. Protein synthesis, posttranslational modifications and aging. Ann NY Acad Sci 1992; 663:48-62.

89. Mays-Hoopes LL. Macromolecular methylation during aging. Rev Biol Res Aging 1985; 2:361-93.

90. McFadden PN, Clarke S. Protein carboxyl methyltransferase and methyl acceptor proteins in aging and cataractus tissue of the human eye lens. Mech Ageing Develop 1986; 34:91-105.

91. Sellinger OZ, Kramer CM, Conger A et al. The carboxylmethylation of cerebral membrane-bound proteins increases with age. Mech Ageing Develop 1988; 43:161-73.

92. Kay MMB. Molecular aging of membrane molecules and cellular removal. In: Goldstein AL, editor. Biomedical Advances in Aging. New York: Plenum Press; 1990. p. 147-61.

93. Porter MB, Pereira-Smith OM, Smith JR. Common senescent cell-specific antibody epitopes on fibronectin in species and cells of varied origin. J Cell Physiol 1992; 150:545-51.

94. Hébert L, Pandey S, Wang E. Commitment to cell death is signaled by the appearance of a terminin protein of $30 \mathrm{kDa}$. Exp Cell Res 1994; 210:10-8.

95. Selkoe DJ. Aging brain, aging mind. Sci Amer 1992; 267:135-42.

96. Esler WP, Wolfe MS. A portrait of Alzheimer secretases- new features and familiar faces. Science 2001; 293:1449-54.

97. DiPaolo BR, Pignolo RJ, Cristofalo VJ. Overexpression of the two-chain form of cathepsin B in senescent WI-38 cells. Exp Cell Res 1992; 201:500-5.

98. Sottile J, Mann DM, Diemer V et al. Regulation of collagenase and collagenase mRNA production in early- and late-passage human diploid fibroblasts. J Cell Physiol 1989; 138:281-90.

99. Whiteheart SW, Shenbagamurthi P, Chen L et al. Murine elongation factor 1a (EF-1a) is posttranslationally modified by novel amide-linked ethanolamine-phosphoglycerol moieties. J Biol Chem 1989; 264:14334-41. 
100. Park MH, Wolff EC, Folk JE. Is hypusine essential for eukaryotic cell proliferation? TIBS 1993; 18:475-9.

101. Nagaraj RH, Sell DR, Prabhakaram M et al. High correlation between pentosidine protein crosslinks and pigmentation implicates ascorbate oxidation in human lens senescence and cataractogenesis. Proc Natl Acad Sci USA 1991; 88:10257-61.

102. Norsgaard H, Clark BFC, Rattan SIS. Distinction between differentiation and senescence and the absence of increased apoptosis in human keratinocytes undergoing cellular aging in vitro. Exp Gerontol 1996; 31:563-70.

103. Huttner WB. Protein tyrosine sulfation. TIBS 1987; 12:361-3.

104. Marshall CJ. Protein prenylation: a mediator of protein-protein interactions. Science 1993; 259:1865-6.

105. Thelin A, Runquist M, Ericsson J et al. Age-dependent changes in rat liver prenyltransferases. Mech Ageing Dev 1994; 76:165-76.

106. Merker K, Grune T. Proteolysis of oxidised proteins and cellular senescence. Exp Gerontol 2000; 35:779-86.

107. Gaczynska M, Osmulski PA, Ward WF. Caretaker or undertaker? The role of the proteasome in aging. Mech Ageing Dev 2001; 122:235-54.

108. Shringaarpure R, Davies KJA. Protein turnover by the proteasome in aging and disease. Free Rad Biol Med 2002; 32:1084-9.

109. Pan J-X, Short SR, Goff SA et al. Ubiquitin pools, ubiquitin mRNA levels and ubiquitin-mediated proteolysis in aging human fibroblasts. Exp Gerontol 1993; 28:39-49.

110. Bulteau AL, Petropoulos I, Friguet B. Age-related alterations of proteasome structure and function in aging epidermis. Exp Gerontol 2000; 35:767-77.

111. Brégégére F, Milner Y, Friguet $B$. The ubiquitin-proteasome system at the crossroads of stress-response and ageing pathways: a handle for skin care? Aging Res Rev 2006; 5:60-90.

112. Carrard G, Bulteau AL, Petropoulos I et al. Impairment of proteasome structure and function in aging. Int J Biochem Cell Biol 2002; 34:1461-74.

113. Terman A, Kurz T, Gustafsson B et al. Lysosomal labilization. IUBMB LIfe 2006; 58:531-9.

114. Terman A, Gustafsson B, Brunk UT. Autophagy, organelles and ageing. J Pathol 2007; 211:134-43.

115. Wick M, Bürger $\mathrm{C}$, Brüsselbach $S$ et al. A novel member of human tissue inhibitor of metalloproteinases (TIMP) gene family is regulated during G1 progression, mitogenic stimulation, differentiation and senescence. J Biol Chem 1994; 269:18953-60.

116. Hearn MG, Edland SD, Ogburn CE et al. Trypsin inhibitor activities of fibroblasts increase with age of donor and are unaltered in familial Alzheimer's disease. Exp Gerontol 1994; 29:611-23.

117. Rattan SIS. Theories of biological aging: genes, proteins and free radicals. Free Rad Res 2006; 40:1230-8.

118. Rattan SIS. Increased molecular damage and heterogeneity as the basis of aging. Biol Chem 2008; 389:267-72. 